\title{
Fast magnetic domain-wall motion in a ring-shaped nanowire driven by a voltage
}

\author{
Jia-Mian $\mathrm{Hu}^{1 \dagger}$, Tiannan Yang ${ }^{1 \dagger}$, Kasra Momeni ${ }^{1}$, Xiaoxing Cheng ${ }^{1}$, Lei Chen ${ }^{1}$, Shiming Lei $^{1}$, Shujun \\ Zhang $^{1}$, Susan Trolier-McKinstry ${ }^{1}$, Venkatraman Gopalan ${ }^{1}$, Gregory P. Carman ${ }^{2}$, Ce-Wen Nan ${ }^{3}$, and \\ Long-Qing Chen ${ }^{1}$ \\ ${ }^{1}$ Department of Materials Science and Engineering, Pennsylvania State University, \\ University Park, Pennsylvania 16802, USA \\ ${ }^{2}$ Department of Mechanical and Aerospace Engineering, University of California, Los Angeles, \\ California 90095, USA \\ ${ }^{3}$ School of Materials Science and Engineering, and State Key Lab of New Ceramics and Fine \\ Processing, Tsinghua University, Beijing 100084, China
}

\section{Summary of Supplementary Figures}

Figure S1 Non-uniform strain distribution in the magnetic ring

Figure S2 Architecture of potential devices based on the proposed design

Figure S3 Analytical model of strain-driven magnetic domain-wall motion in a magnetic ring Figure S4 Origin of the incubation delay

Figure S5 Evidence of enhanced structural stability with increasing strains Figure S6 Highest strain-induced mobility in in-plane transverse magnetic domain-walls 


\section{Supplementary Figure S1}
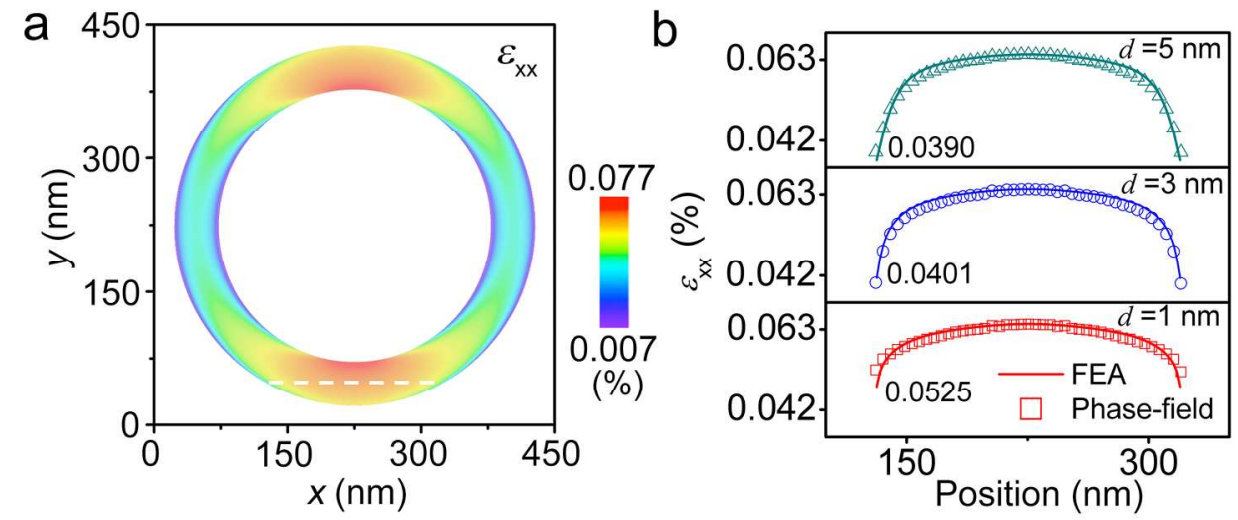

Figure S1. a, Distribution of strain $\varepsilon_{x x}$ at mechanical equilibrium when applying a uniaxial strain of $0.0766 \%$ to the amorphous $\mathrm{Co}_{40} \mathrm{Fe}_{40} \mathrm{~B}_{20}$ ring along the $x$ (i.e., [100] axis) direction, obtained through phase-field simulations. b. Thickness-dependent (from top, $d=5 \mathrm{~nm}$, to bottom, $d=1 \mathrm{~nm}$ ) strain profiles along the center transect of the highly strained region (see the dashed line in a) calculated from phasefield simulations, is consistent with the results obtained through finite element analyses (FEA). 


\section{Supplementary Figure S2}
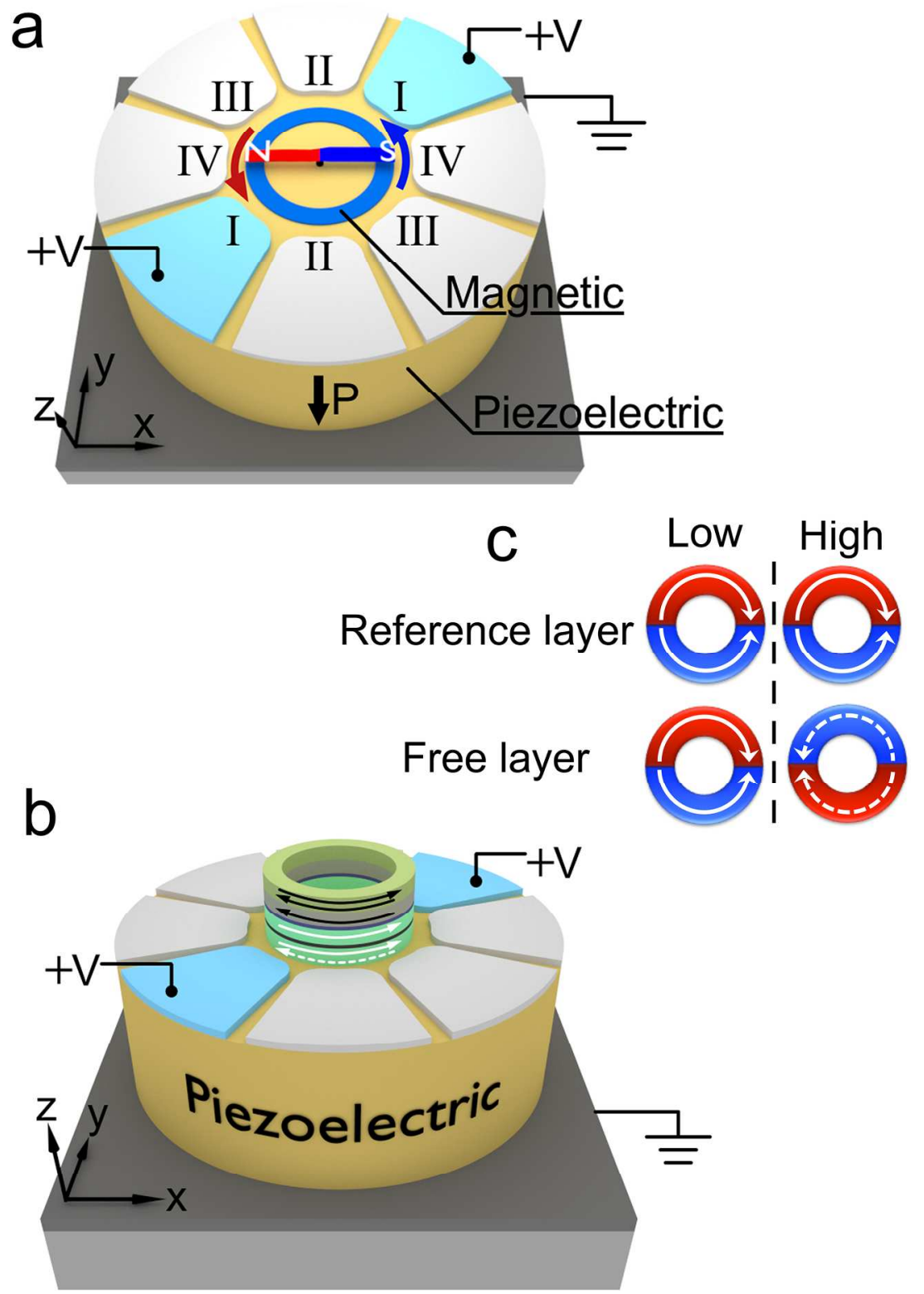

Figure S2. a, Schematics of a nanoscale magnetoelectric motor. Magnetic domain-wall motion changes the orientation of the magnetic stray field outside the ring, and makes the rotor magnet spin. b, Schematic of a ring-based magnetoelectric random access memory. The ring-shaped magnetoresistive multilayer consisting of (from top to bottom) an antiferromagnetic pinning layer, a synthetic antiferromagnetic tri-layer with a pinned top layer and a reference bottom layer separated by an ultrathin non-magnetic spacer layer, a tunnel barrier layer, and a free layer. The arrows indicate magnetization orientations. c, Schematics showing that a $180^{\circ}$ motion of the two domain-walls in the ring-shaped free layer can reverse the net magnetization, which would become parallel or antiparallel to the net magnetization in the ring-shaped reference layer such that low or high electric resistance states can be obtained, respectively. 


\section{Supplementary Figure S3}
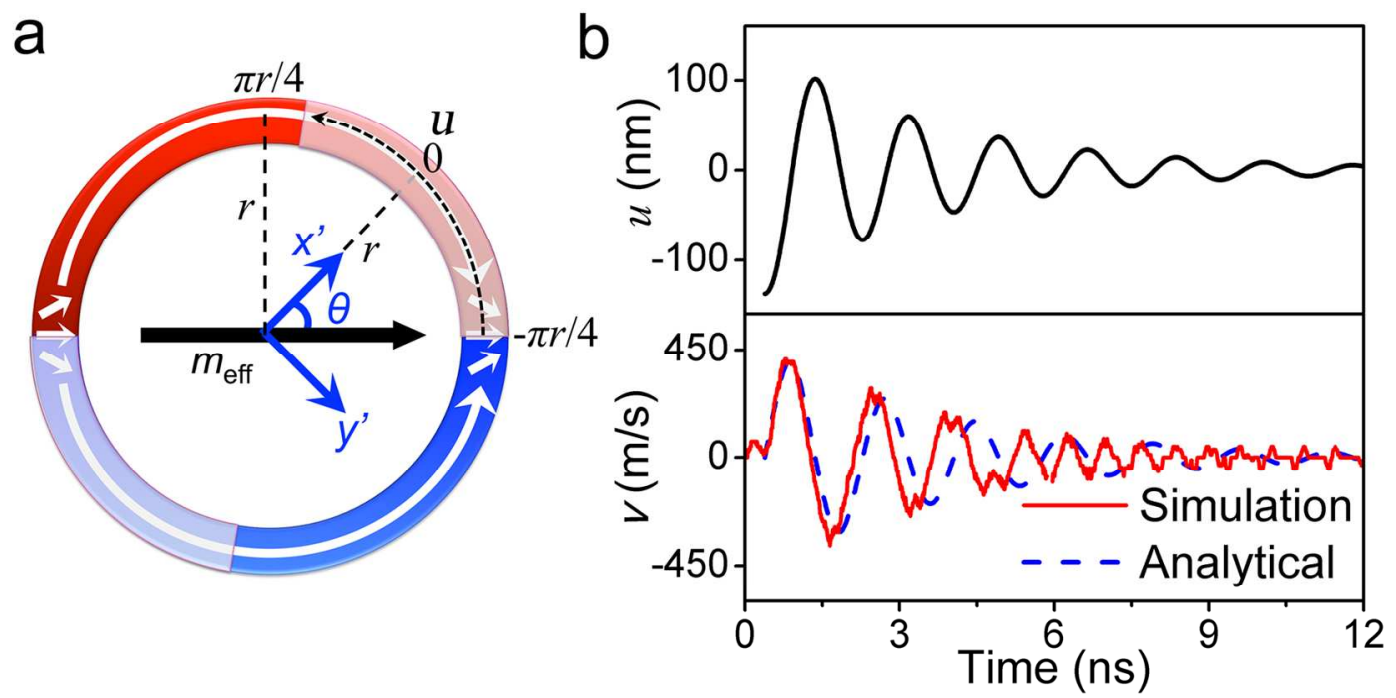

Figure S3. a, Schematics of the effective magnetization vector $m_{\mathrm{eff}}$, rotated frame of biaxial axes $x$ ' and $y$ ' for the application of piezostrains, and the instantaneous displacement of domain-walls $u(t)$ along the magnetic ring within the shaded range. b. Instantaneous domain-wall displacement $u(t)$ and velocity $v(t)$ calculated through equation (S4), with comparison to the $v(t)$ obtained from phase-field simulations (see figure $3 \mathrm{a}$ in the main text). 


\section{Supplementary Figure S4}
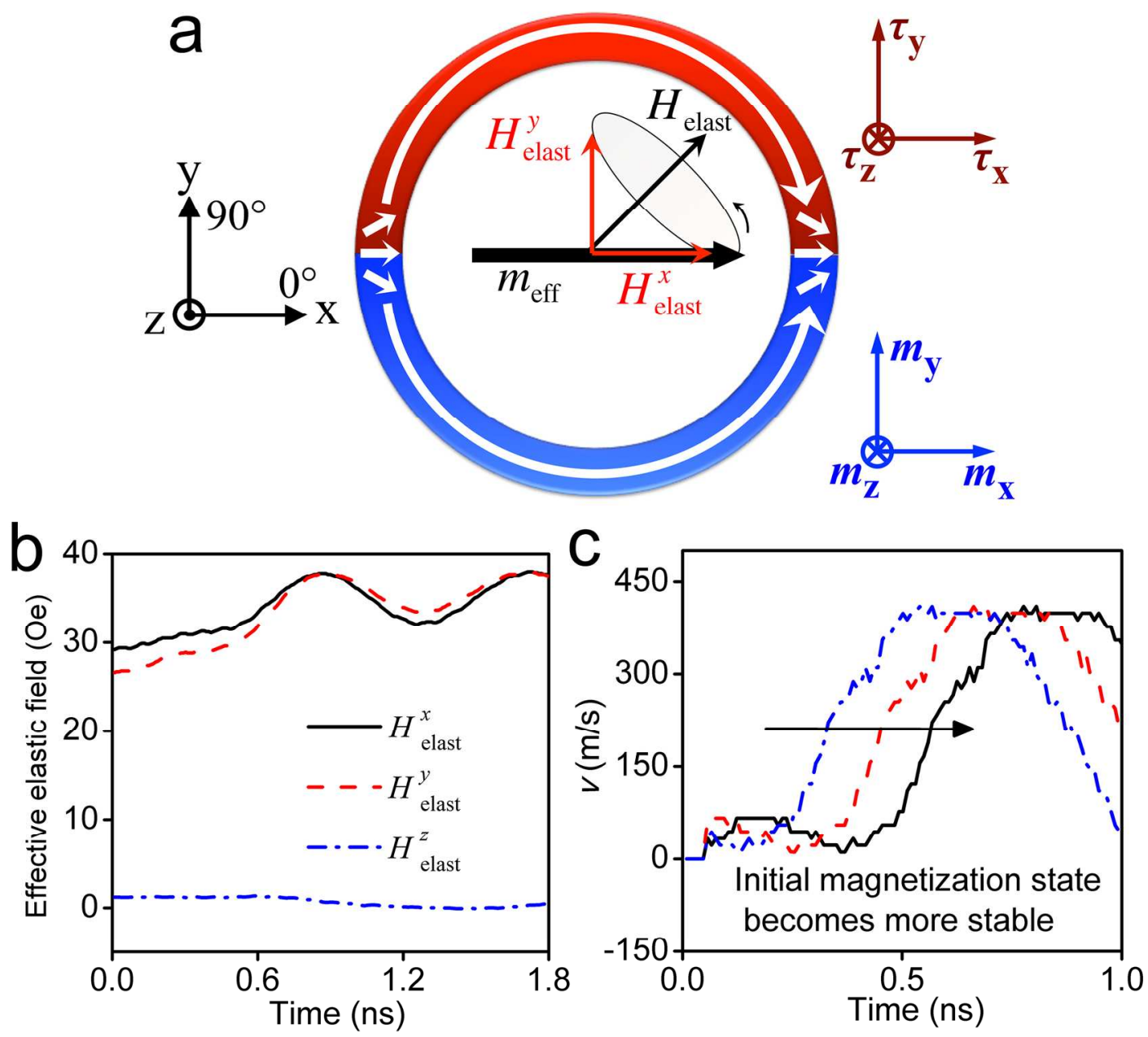

Figure S4. a, Schematics showing the orientations of the net magnetization components $\left(m_{\mathrm{x}}, m_{\mathrm{y}}, m_{\mathrm{z}}\right)$ at the initial state, the effective magnetic field from elastic anisotropy energy $\left(\boldsymbol{H}_{\text {elast }}\right.$, denoted as effective elastic field) upon applying a static uniaxial piezostrain of $0.0766 \%$ along the [110] axis of the amorphous $\mathrm{CoFeB}$ ring, and the torques $\left(\tau_{\mathrm{x}}, \tau_{\mathrm{y}}, \tau_{\mathrm{z}}\right)$ generated by $\boldsymbol{H}_{\text {elast. }}$ Note that $\tau_{\mathrm{x}}=m_{\mathrm{z}} \times H_{\text {elast }}^{y}, \tau_{\mathrm{y}}=m_{\mathrm{z}} \times$ $H_{\text {elast }}^{x}$, and $\tau_{\mathrm{z}}=\left(m_{\mathrm{x}} \times H_{\text {elast }}^{y}\right)+\left(m_{\mathrm{y}} \times H_{\text {elast }}^{x}\right)$. The effective magnetization vector would precess around the $\boldsymbol{H}_{\text {elast }}$ in an anticlockwise manner, resulting from the oscillatory, to-and-fro motion of the two in-plane transverse domain-walls. b. Time-dependent evolution of the components of $\boldsymbol{H}_{\text {elast }}$ corresponding to the applied uniaxial piezostrains. c, Instantaneous domain-wall velocities simulated under the same applied piezostrain of $0.0766 \%$ but with initial magnetization states becoming more stable, specifically, with the magnitude of $\boldsymbol{m}_{\mathrm{z}}$ decreasing from about -0.00233 (dashed dotted line), to -0.00104 (dashed line), then to -0.00065 (solid lines). 


\section{Supplementary Figure S5}
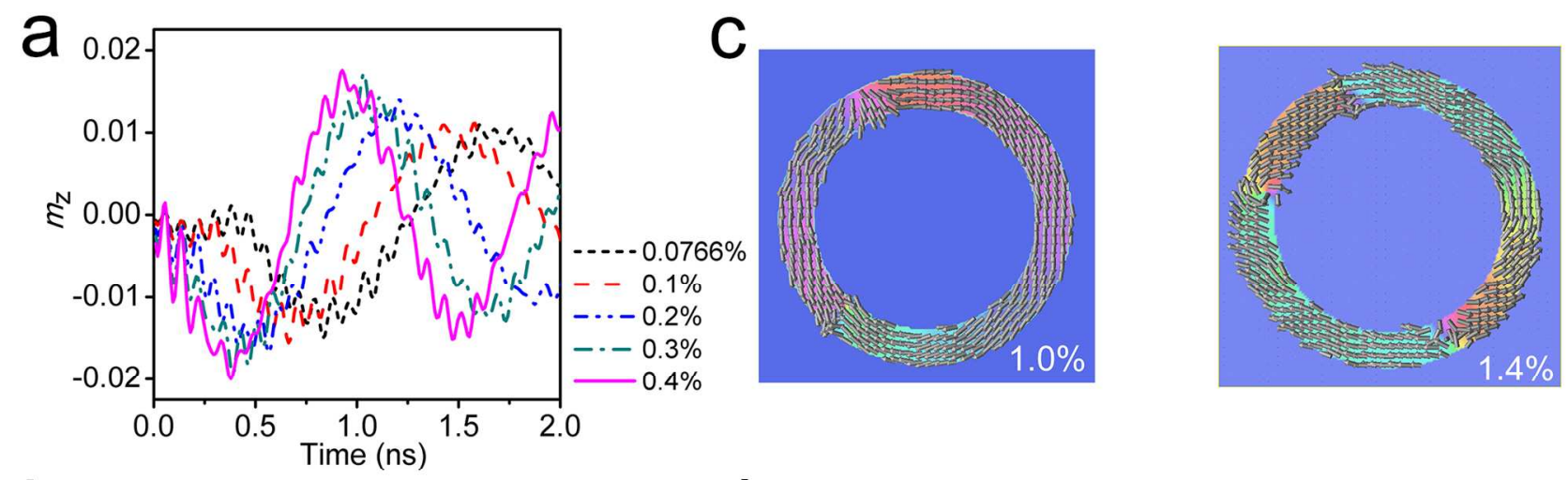

b
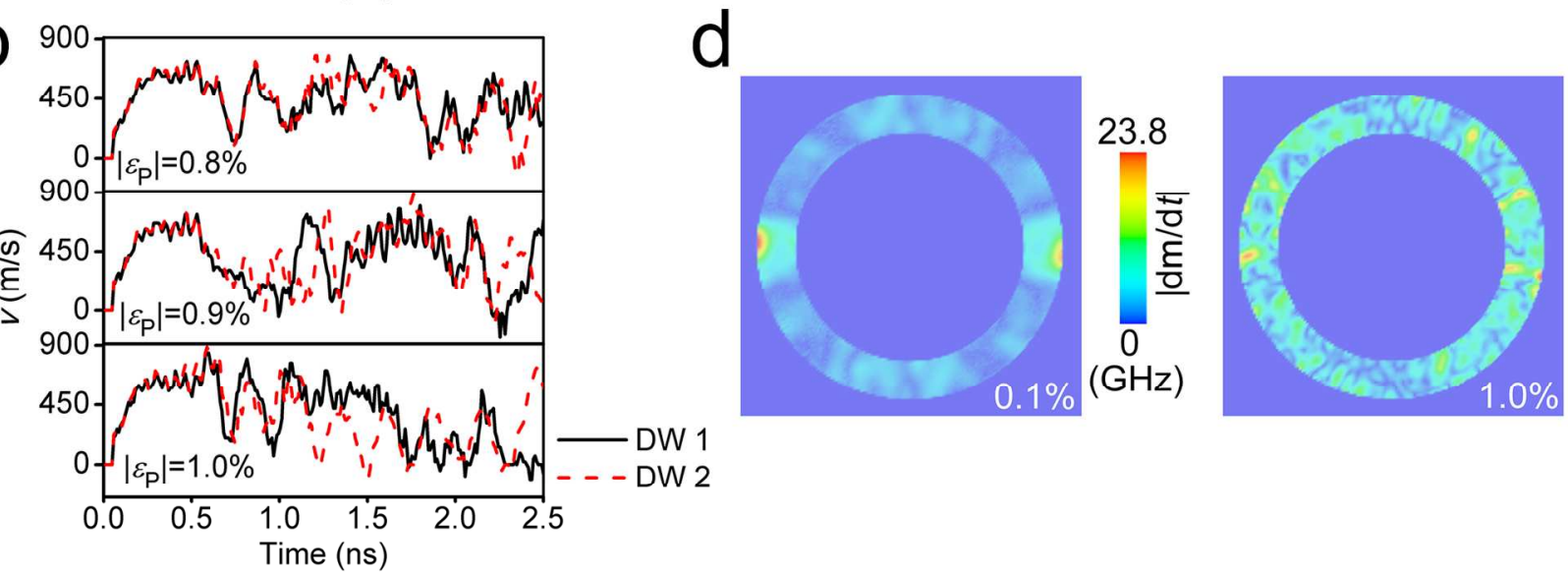

89.8

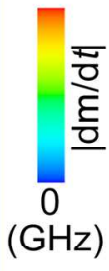

Figure S5. a, Time-dependent evolution of the out-of-plane net magnetization component $m_{\mathrm{z}}$ under different strains. b. Instantaneous magnetic domain-wall velocities during one full $360^{\circ}$ cycle when the magnitude of strain pulses are $0.8 \%, 0.9 \%$, and $1.0 \%$. c, Formation of asymmetric onion-domain (left) and in-plane vortex domain-wall (right) under larger strains of $1.0 \%$ and $1.4 \%$, respectively. d, Distribution of the magnitude of the instantaneous torque $|d \boldsymbol{m} / d t|$ under the strain of $0.1 \%$ and $1 \%$. 


\section{Supplementary Figure S6}
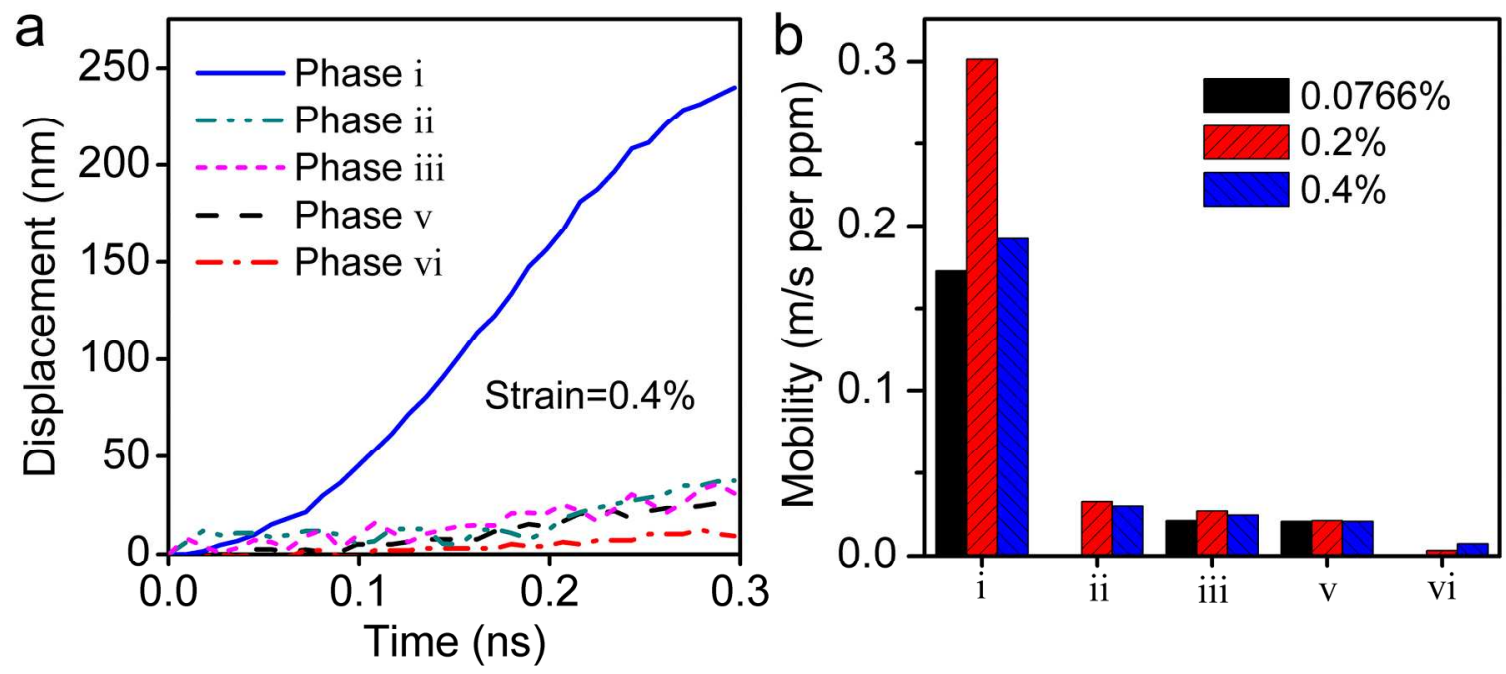

Figure S6. a, Time-dependent displacement of the five representative domain-walls shown in Figure 4 in the main text, and $\mathbf{b}$, their corresponding mobility under different applied strains. Phase $\mathrm{i}$ (in-plane transverse domain-walls): $300 \mathrm{~nm}$ (inner diameter) $\times 400 \mathrm{~nm}$ (outer diameter) $\times 5 \mathrm{~nm}$ (thickness); phase ii $(300 \mathrm{~nm} \times 500 \mathrm{~nm} \times 30 \mathrm{~nm})$; phase iii $(300 \mathrm{~nm} \times 550 \mathrm{~nm} \times 45 \mathrm{~nm})$; phase v $(300 \mathrm{~nm} \times 325 \mathrm{~nm} \times 30$ $\mathrm{nm})$; phase vi $(300 \mathrm{~nm} \times 350 \mathrm{~nm} \times 80 \mathrm{~nm})$. Phase iv (not shown) represents the multiple vortices structure (see Figure $4 \mathrm{a}$ in the main text) in which case it is difficult (and also meaningless) to track the position of a certain vortex. 


\section{Supplementary Note S1}

The two terms on the left of equation (2) can be obtained directly by taking time derivative on the two terms on the left of equation (1). The key is thus to generate an analytical expression of the elastic energy term [i.e., on the right of equation (1)] for such a ring with non-uniform magnetization distribution. Given that the motion of the two transverse domains can approximately be considered as a coherent rotation of an in-plane effective magnetization vector below the critical strain $\left|\varepsilon_{\text {cr }}\right|$, one may have $m_{\mathrm{x}}=k \cos \theta, m_{\mathrm{y}}{ }^{\prime}=k \sin \theta$, and $m_{\mathrm{z}}=0$ in a rotated frame $x^{\prime} y^{\prime}$ shown in Figure S3a. The corresponding elastic energy density $f_{\text {elast }}$ can therefore be written as,

$$
\begin{aligned}
& f_{\text {elast }}=\frac{1}{2} c_{11}\left[\left(\varepsilon_{x^{\prime} x^{\prime}}-\varepsilon_{x^{\prime} x^{\prime}}^{0}\right)^{2}+\left(\varepsilon_{y y^{\prime}}-\varepsilon_{y y^{\prime}}^{0}\right)^{2}+\left(\varepsilon_{z^{\prime} z^{\prime}}-\varepsilon_{z^{\prime} z^{\prime}}^{0}\right)^{2}\right] \\
& +c_{12}\left[\left(\varepsilon_{x^{\prime} x^{\prime}}-\varepsilon_{x^{\prime} x^{\prime}}^{0}\right)\left(\varepsilon_{y^{\prime} y^{\prime}}-\varepsilon_{y^{\prime} y^{\prime}}^{0}\right)+\left(\varepsilon_{x^{\prime} x^{\prime}}-\varepsilon_{x^{\prime} x^{\prime}}^{0}\right)\left(\varepsilon_{z^{\prime} z^{\prime}}-\varepsilon_{z^{\prime} z^{\prime}}^{0}\right)+\left(\varepsilon_{y y^{\prime}}-\varepsilon_{y y^{\prime}}^{0}\right)\left(\varepsilon_{z^{\prime} z^{\prime}}-\varepsilon_{z^{\prime} z^{\prime}}^{0}\right)\right] \\
& +2 c_{44}\left[\left(\varepsilon_{x^{\prime} y^{\prime}}-\varepsilon_{x^{\prime} y^{\prime}}^{0}\right)^{2}+\left(\varepsilon_{y z^{\prime}}-\varepsilon_{y^{\prime} z^{\prime}}^{0}\right)^{2}+\left(\varepsilon_{x z^{\prime}}-\varepsilon_{x^{\prime} z^{\prime}}^{0}\right)^{2}\right],
\end{aligned}
$$

where $\varepsilon_{x^{\prime} x^{\prime}}$ and $\varepsilon_{y^{\prime} y^{\prime}}$ are the total biaxial in-plane anisotropic piezostrains applied onto the ring and the shear strains $\left(\varepsilon_{x y^{\prime}}, \varepsilon_{y^{\prime} z^{\prime}}\right.$, and $\left.\varepsilon_{x^{\prime} z^{\prime}}\right)$ are all zero in the rotated frame, with the stress-free strain $\varepsilon_{i j}^{0}$ $=1.5 \lambda_{\mathrm{s}}\left(m_{i} m_{j}-1 / 3\right)$ and $1.5 \lambda_{\mathrm{s}} m_{i} m_{j}$ for $i=j$ and $i \neq j$, respectively $\left(i, j=x^{\prime}, y^{\prime}, z^{\prime}\right)$, and with the elastic stiffness constants $c_{44}=0.5\left(c_{11}-c_{22}\right)$ for an amorphous magnet. We neglect the contribution from the heterogeneous strains in the present analytical model for simplicity, i.e., $\varepsilon_{x^{\prime} x^{\prime}} \approx \overline{\varepsilon_{x^{\prime} x^{\prime}}} \varepsilon_{y^{\prime} y^{\prime}} \approx \overline{\varepsilon_{y^{\prime} y^{\prime}}}$, and so forth.

Consider a static piezostrain $\varepsilon_{\mathrm{p}}\left[\approx\left(\varepsilon_{x^{\prime} x^{\prime}}-\varepsilon_{y^{\prime} y^{\prime}}\right) /(1-\eta)\right]$ of $0.0766 \%$ applied along the biaxial $x^{\prime}$ and $\mathrm{y}^{\prime}$ axes, where $\eta$ is the degree of strain relaxation $(\sim 30 \%)$. In this case, the only time-dependent parameter in equation (S1) (through $m_{\mathrm{x}}$, and $m_{\mathrm{y}}$ ) is $\theta$, i.e., $\theta(t)=u(t) / r$. Here $u$ refers to the displacement of the domain-wall along the ring, and $r$ is the radius of the ring $(=175 \mathrm{~nm})$. The shades in figure S3a roughly mark the spatial range of the oscillatory, back-and-forth wall motion around the $x$ '-axis, and $u$ is set to 
zero at the intersection of the axis of $\varepsilon_{\mathrm{p}}$ and the ring where $\theta(t=0)=45^{\circ}$. Removing all terms that are independent on $\theta$ in, equation (S1) reduces to,

$$
f_{\text {elast }}(t)=-\frac{3}{4} k^{2} \lambda_{s}\left(c_{11}-c_{12}\right)(1-\eta) \varepsilon_{\mathrm{p}} \cos \left(\frac{2 u(t)}{r}\right)
$$

A time derivative of the term $\left[f_{\text {elast }}(t=0)-f_{\text {elast }}(t)\right]$ thus yields,

$$
-\frac{d f_{\text {elast }}(t)}{d t}=-\frac{3 k^{2} \lambda_{s}}{2 r}\left(c_{11}-c_{12}\right)(1-\eta) \varepsilon_{\mathrm{p}} \sin \left(\frac{2 u(t)}{r}\right) .
$$

Multiplied by the volume of the ring $V_{\text {ring }}$, equation (S3) will be the driving force for the magnetic domain-wall motion (in the unit of Newton). Given that the instantaneous velocity $v(t)=d u(t) / d t$, equation (2) can be further rewritten as,

$$
m^{*} \frac{d^{2} u(t)}{d t^{2}}+\beta \frac{d u(t)}{d t}=-\frac{3 k^{2} \lambda_{s} V_{\text {ring }}}{2 r}\left(c_{11}-c_{12}\right)(1-\eta) \varepsilon_{\mathrm{p}} \sin \left(\frac{2 u(t)}{r}\right)
$$

A numerical solution of equation (S4) under the initial conditions of $u(t=0)=-\pi r / 4 \approx-137.4 \mathrm{~nm}$ (see figure S3a) and $v(t=0)=d u(t=0) / d t=0 \mathrm{~m} / \mathrm{s}$ allows us to calculate both $u(t)$ and $v(t)$, as shown by the top and bottom panel of figure $\mathrm{S} 3 \mathrm{~b}$, respectively. In particular, after subtracting the incubation delay $(\sim 0.38$ ns, see Supplementary Note S2), the $v(t)$ calculated through equation (S4) is generally consistent with the simulated $v(t)$ in both the amplitude and frequency of oscillation, notably in the first period (figure $\mathrm{S} 3 \mathrm{~b})$. This is important to the calculation of the oscillating $v(t)$ during the full $360^{\circ}$ unidirectional motion and hence the average velocity (see figure $3 \mathrm{c}$ in the main text) under pulse drive, because the orientation of the applied piezostrain $\left(\varepsilon_{\mathrm{p}}\right)$ would always be changed within the very first period. In the present analytical model, the $u(t)$ during a full $360^{\circ}$ unidirectional domain-wall motion cycle is solved by varying the orientation of $\varepsilon_{\mathrm{p}}$, and the initial conditions of $u(t)$ and $v(t)$ during each one of the eight stages (see figure $2 b)$. 


\section{Supplementary Note S2}

After being poled in a strong static magnetic field, the CoFeB ring shows an onion-type magnetic domain with two in-plane transverse domain-walls, with the net magnetization components $m_{\mathrm{x}} \approx 0.72$, $m_{\mathrm{y}} \approx 0.0013$, and $m_{\mathrm{z}} \approx-6.5 \times 10^{-4}$. This is the initial magnetization state in our simulations. A piezostrain applying along the $x y$-axis $\left(45^{\circ} / 225^{\circ}\right.$, see Figure S4a) is equivalent to an effective magnetic field along the same axis from elastic anisotropy energy (denoted as $\boldsymbol{H}_{\text {elast }}$ ), which is further equivalent to effective magnetic fields along the $x$ and $y$ axes (denoted as $H_{\text {elast }}^{x}$ and $H_{\text {elast }}^{y}$ ), as shown in Figure S4a. $H_{\text {elast }}^{x}$ and $H_{\text {elast }}^{y}$ can be numerically calculated from the phase-field model. Figure $\mathrm{S} 4 \mathrm{~b}$ shows the time-dependent evolution of the $H_{\text {elast }}^{x}$ and $H_{\text {elast }}^{y}$ when applying a uniaxial piezostrain of $0.076 \%$ along the $45^{\circ} / 225^{\circ}$. The positive $H_{\text {elast }}^{x}$ and $H_{\text {elast }}^{y}$ indicate that these fields are along the $+x\left(0^{\circ}\right)$ and $+y\left(90^{\circ}\right)$ axes,

respectively. It is worth noting that the in-plane torque $\tau_{\mathrm{y}}=\left(m_{z} \times H_{\text {elast }}^{x}\right)$ (see Figure S4a), which is perpendicular to initial direction of the effective magnetization vector ( $\left.m_{\mathrm{eff}}\right)$, plays an important role in initiating the precession of $m_{\text {eff }}$ around the $\boldsymbol{H}_{\text {elast }}$.

As the magnitude of $m_{\mathrm{z}}$ is quite small (almost zero) at the initial state, a certain period of time (termed the incubation delay) is needed at the initial stage such that $m_{\mathrm{z}}$ can increase through fluctuations to provide the necessary 'start-up' torque. In an extreme case, if $m_{z}$ were precisely zero at the initial state (which is impossible to specify in numerical simulations and also impractical in experiments), $m_{\text {eff }}$ would not be able to precess and the magnetic domain-walls would not move. As shown in Figure S4c, when the initial magnetic state becomes more stable (i.e., $m_{\mathrm{z}}$ decreases), longer incubation delay at the initial stage appears upon applying the same piezostrain of $0.0766 \%$. Accordingly, it takes longer time for the instantaneous velocity $v(t)$ to reach its peak value. 


\section{Supplementary Note S3}

Given that the out-of-plane torque $\left(\tau_{\mathrm{z}}\right)$ generated by the effective elastic field $\left(\boldsymbol{H}_{\text {elast }}\right)$ can be expressed as $\tau_{\mathrm{z}}=\left(m_{\mathrm{x}} \times H_{\text {elast }}^{y}\right)+\left(m_{\mathrm{y}} \times H_{\text {elast }}^{x}\right)$ (see figure S4a), applying larger strains would increase the $\tau_{\mathrm{z}}$ and hence the out-of-plane net magnetization component $\left(m_{\mathrm{z}}\right)$, as shown in Figure S5a.

Under strains larger than $0.4 \%$, the magnetization vectors inside the magnetic domains start to show appreciable precession around the $\boldsymbol{H}_{\text {elast }}$ due to the significantly enhanced, spatially dispersive torques (figure S5b), which impedes the propagation of magnetic domain-wall(s) and further makes the motion of the two domain-walls asynchronous. For illustration, figure S5b shows the spatial distributions of the magnitude of the instantaneous torque $|\mathrm{d} m / \mathrm{d} t|$ under the strains of $0.1 \%$ and $1 \%$ that yield equal average domain-wall velocities in a full $360^{\circ}$ domain-wall cycle. In the case of $0.1 \%$, the torques show intensification close to the outer edges of the two domain-walls, while they are negligible in the remaining regions, indicating almost immobile magnetization vectors in the domains. In contrast, spatially dispersive torque distribution, albeit with stronger magnitude (see the color bars), is exhibited in the case of $1 \%$. 


\section{Supplementary Note S4}

As shown in Figure S6a, upon applying the same strain of $0.4 \%$, the in-plane transverse domainwalls (phase i) propagate the fastest (see the sharpest increase in the displacement), while the out-ofplane vortex domain-wall (phase vi) propagates the slowest. There are no appreciable differences in speed in the cases of the in-plane single- (phase ii), double-vortex (phase iii) domain-walls, and the outof-plane transverse domain-walls (phase v). Figure S6b further lists the mobility of the five representative domain-wall structures under different strains, among which the in-plane transverse domain-wall shows the highest mobility.

The in-plane transverse domain-walls show the highest mobility in response to strain because the local magnetization inside the walls does not fully cancel out (as in the case of vortex walls). Accordingly, a substantial amount of torque $\left(\boldsymbol{m} \times \boldsymbol{H}_{\text {elast }}\right)$ can be produced inside the magnetic domainwalls. By comparison, no net magnetization is exhibited in the case of out-of-plane transverse domainwalls because of the different polarity in the two domain-walls (e.g., pointing up and down, see Figure 4c). Thus, the magnetization torque is small.

The highest mobility in the case of in-plane transverse domain-walls also results from the smallest degree of strain relaxation among the six specific dimensions (see Figure 4a). In contrast, the out-ofplane vortex domain-walls not only possess local magnetization vectors that are almost fully cancelled out, but also the highest degree of strain relaxation, resulting in the lowest strain-induced domain-wall mobility. 


\section{Supplementary Note S5}

The main motivation for utilizing a voltage to drive magnetic domain-wall is to fundamentally solve the overheating issue brought by the use of a current (via spin-torques) in miniaturized devices. In a recent report, a magnetic domain-wall velocity up to $500 \mathrm{~m} / \mathrm{s}$ has been achieved in an arc-shaped nanowire with a vertical current pulse injected through a multilayered magnetic tunnel junction (MTJ) on top of the nanowire. ${ }^{1}$ As stated in ref. 1 , it is possible to achieve a sub-mA critical current in a well-optimized device design that has a dimension below $100 \mathrm{~nm}$. As an optimistic estimate, the heat dissipation can be reduced down to about $0.16 \mathrm{pJ}$. This estimate is based on a critical current of $0.1 \mathrm{~mA}$, a characteristic junction resistance on the order of $5 \mathrm{k} \Omega$ for sub-100 nm MTJs, ${ }^{2}$ a current pulse duration of $3.2 \mathrm{~ns}$ during which the domain-wall can propagate for the same distance $(\sim 550 \mathrm{~nm})$ and at the same average velocity $(\sim 170 \mathrm{~m} / \mathrm{s})$ as here. In a more recent work ${ }^{3}$ where magnetic domain-wall velocity up to $750 \mathrm{~m} / \mathrm{s}$ has been achieved in nanowires made up of synthetic antiferromagnets with a current density of about 300 $\mathrm{MA} / \mathrm{cm}^{2}$, where the spin Hall effect is utilized to apply spin-transfer-torque to the magnetic domainwalls. The work also reports a domain-wall velocity of about $170 \mathrm{~m} / \mathrm{s}$ with a current density of about 90 $\mathrm{MA} / \mathrm{cm}^{2}$ in an optimized nanowire (approximately 5-nm-thick, $2-\mu \mathrm{m}$-wide). ${ }^{3}$ By scaling down the width of the nanowire to $50 \mathrm{~nm}$ (the same as here), the current decreases to about $0.2 \mathrm{~mA}$. An ideal heat dissipation of about $6 \mathrm{pJ}$ is further estimated based on the 3.2-ns pulse duration and a nanowire resistance of about $40 \mathrm{k} \Omega$. This resistance value of the $50-\mathrm{nm}$-wide nanowire is 40 times larger than the $1 \mathrm{k} \Omega$ of the $2-\mu \mathrm{m}$-wide nanowire of the same thickness (the latter value is given in the Supplemental Materials of ref. ${ }^{3}$ ). 


\section{Supplementary Note S6}

For an out-of-plane poled polycrystalline PZT disk with $\infty \mathrm{m}$ symmetry, the matrices of $\boldsymbol{e}, \boldsymbol{\varepsilon}_{\mathrm{r}}$, and $\boldsymbol{c}_{E}$ used for finite-element simulations are the same as those given in ref. 4,

$$
\begin{aligned}
& c_{E}=\left[\begin{array}{cccccc}
127.21 & 80.21 & 84.67 & 0 & 0 & 0 \\
& 127.21 & 84.67 & 0 & 0 & 0 \\
& & 117.43 & 0 & 0 & 0 \\
& & & 22.99 & 0 & 0 \\
& & & & 22.99 & 0 \\
& & & & 23.47
\end{array}\right] \mathrm{GPa} ; \\
& e=\left[\begin{array}{cccccc}
0 & 0 & 0 & 0 & 17.0345 & 0 \\
0 & 0 & 0 & 17.0345 & 0 & 0 \\
-6.62281 & -6.62281 & 23.2403 & 0 & 0 & 0
\end{array}\right] \mathrm{C} / \mathrm{m}^{2} ; \\
& \varepsilon_{r}=\left[\begin{array}{ccc}
1704.4 & 0 & 0 \\
0 & 1704.4 & 0 \\
0 & 0 & 1433.6
\end{array}\right] .
\end{aligned}
$$

In experimental, bilayered $\mathrm{Ti} / \mathrm{Au}$ electrodes can be deposited on top polycrystalline $\mathrm{PZT},{ }^{5}$ where the Ti bottom layer and the top Au layer are utilized to promote adhesion and electric conductivity, respectively. Given that the Ti layer is usually considerably thinner than Au, here we utilize the parameters of pure Au in the finite-element model for simplicity. The polycrystalline Au is treated as isotropic, and has a $\varepsilon_{\mathrm{r}}$ of 6.9, a Poisson's ratio $v$ of 0.42 , and a Young's modulus $Y$ of $79 \mathrm{GPa}$, all taken from the materials libraries of the commercial COMSOL Multiphysics ${ }^{\circledR}$ software. 


\section{References in Supplemental Materials:}

1. Metaxas, P. J.; Sampaio, J.; Chanthbouala, A.; Matsumoto, R.; Anane, A.; Fert, A.; Zvezdin, K. A.; Yakushiji, K.; Kubota, H.; Fukushima, A.; Yuasa, S.; Nishimura, K.; Nagamine, Y.; Maehara, H.; Tsunekawa, K.; Cros, V.; Grollier, J. Sci. Rep. 2013, 3, 1829.

2. Ikeda, S.; Miura, K.; Yamamoto, H.; Mizunuma, K.; Gan, H. D.; Endo, M.; Kanai, S.; Hayakawa, J.; Matsukura, F.; Ohno, H. Nat. Mater. 2010, 9, (9), 721-724.

3. Yang, S.-H.; Ryu, K.-S.; Parkin, S. Nat. Nanotechnol. 2015, 10, (3), 221-226.

4. Liang, C.-Y.; Keller, S. M.; Sepulveda, A. E.; Sun, W.-Y.; Cui, J.; Lynch, C. S.; Carman, G. P. J. Appl. Phys. 2014, 116, (12), 123909

5. Lei, N.; Devolder, T.; Agnus, G.; Aubert, P.; Daniel, L.; Kim, J.-V.; Zhao, W.; Trypiniotis, T.; Cowburn, R. P.; Chappert, C.; Ravelosona, D.; Lecoeur, P. Nat. Commun. 2013, 4, 1378. 